\title{
Application of tuned-mass dampers to the control of floor vibrations
}

\section{Aplicação de tuned-mass dampers para controle de vibrações em lajes}

\author{
G. M. ALMEIDA a \\ mesquita.almeida07@gmail.com \\ C. E. N. MAZZILLI a \\ cenmazzi@usp.br
}

\begin{abstract}
This article proposes a standardized solution for the application of Tuned-Mass Dampers to the control of floor vibrations based on the characteristics of the acting loads associated to human usage and the characteristics of the most common structures of the contemporary engineering practice. In order to simplify its usage by the technical community, the tuning is proposed through the selection of pre-determined components for the assembly of the TMD and the choice of disposition and spacing of the mechanisms. The system efficacy is then verified through the computational case study of a floor before and after the application of the mechanisms.
\end{abstract}

Keywords: tuned-mass damper, TMD, vibrations, floor, comfort.

\section{Resumo}

Este artigo propõe uma solução padronizada de aplicação de Tuned-Mass Dampers (TMD) para controle de vibrações em lajes baseada na análise das características de carregamentos associados à utilização humana e nas características estruturais mais comuns à engenharia contemporânea. De modo a simplificar sua aplicação técnica, a sintonização é proposta por meio da escolha de componentes pré-determinados para a montagem do TMD e pela distribuição e posicionamento dos mecanismos. A eficácia do sistema é então verificada por meio de um estudo computacional de caso de uma laje antes e depois da aplicação dos mecanismos.

Palavras-chave: tuned-mass damper, TMD, vibrações, lajes, conforto. 


\section{Introduction}

Tuned-Mass Dampers, TMD's, are highly efficient systems utilized in the passive control of structural vibrations. They basically consist of an inertial element connected through a damper and a spring to a system to alter its dynamic characteristics. Its operational principle is based on the idea that the TMD resonates in an excitation frequency that coincides to a vibration mode of the structure, thus dissipating the energy that would otherwise act unchecked. This characteristic of the TunedMass Dampers, however, demands that they are tuned to each system, reducing its employability in the contemporary civil engineering practice, which remains reluctant about adopting dynamic concepts in the design of structures. A possibility is thus presented for a standardized solution against recurring vibrational problems to which the applicability of traditional static approaches, such as the increase in stiffness, might be inefficient or unviable. In the case of slab vibrations, the situation is even more critical due to the architectonic demands for larger spans and the ever-increasing use of light partitions, that contribute little to the stiffness and damping of the system unlike the masonry partitions. As such, the development of a set of standardized Tuned-Mass Dampers is proposed, as well as a simplified design guide that will allow the proper tuning for slabs of different sizes, thickness and usages, in a way that facilitates its usage in the contemporary practice.

\subsection{Justification}

According to the AISC Steel Design Guides Series 11: Floor

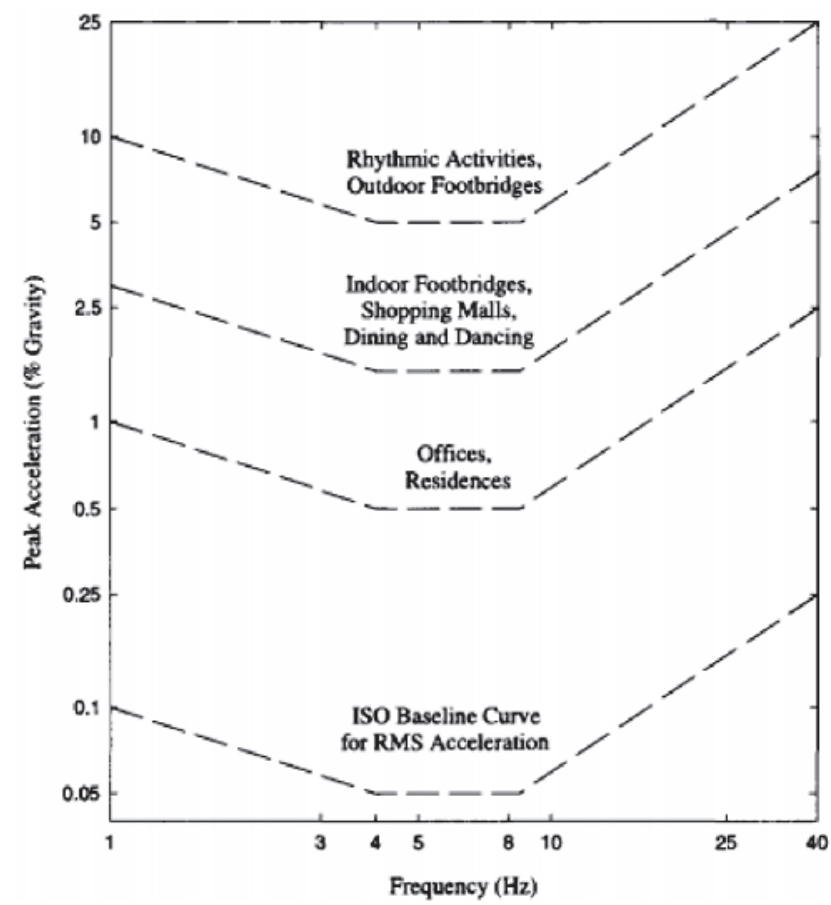

Figure 1

Recommended peak acceleration for human comfort for vibrations due to human activities (AISC Steel Design Guides Series 11; Allen e Murray, 1993; ISO 2631-2: 1989)
Vibrations Due to Human Activity, the human response to floor vibrations is a fairly complex phenomenon that depends, among other factors, of the amplitude of displacements and accelerations one is submitted to, as well as of the current location, user sensitivity and of the nature of the load that originated the vibration. Also, the user reaction is strongly tied to the activities performed in said locations. Users located in residences and offices are troubled by any perceptible vibration, which means any acceleration that surpasses $0,50 \%$ of the gravity, while those located in places in which more energetic activities take place are capable of accepting vibrations of up to $5,00 \%$ of the gravity. Users in intermediary situations, such as dining beside a ballroom or weightlifting beside a fitness class, or even shopping in a shopping center tolerate intermediary values of acceleration, around $1,50 \%$ of the gravity, depending also on the duration of the vibration and the distance from the source. Those limits, however are only valid for vibrations that have their frequencies between $4,0 \mathrm{~Hz}$ and $8,0 \mathrm{~Hz}$, range beyond which users are capable of dealing with greater accelerations.

Throughout the years, many criteria were proposed in order to ascertain the level of human comfort of a set of floors under dynamic effects, being that the AISC divides them in relation to the kind of considered excitation: rhythmic or due to walking. The criteria currently recommended for vibrations due to walking, estimation methods of the required properties and design procedures were initially devised by Allen and Murray, and they differ extensively from the previous analysis based on the "heel-drop test", in which the natural frequencies and damping ratios of the system are ascertained due to the impact created by a person of about $75,0 \mathrm{~kg}$ that stands on his toes, lifting the heels about $60 \mathrm{~mm}$ from the floor and then allow them to hit the floor with full weight. Despite the criteria of Allen and Murray being more complex than the previous, they have a wider range of uses and result in more economic acceptable floors. Using them as basis with a few minor modifications to allow a more embracing application, the Design Guide 11 is based on the dynamic response of a system of floors bore by beams under the loads originated due to walking and can be used to design offices, malls and footbridges, among others. The accelerations are limited in accordance to the recommendations of the International Standards Organization (ISO 2631-2, 1989) adjusted for the intended occupancy. Limits are suggested in terms of root mean square values of acceleration as multiples of the baseline curve presented in Figure [1], which are proposed for offices, malls and indoors footbridges, and outdoors footbridges, defined by multiplying the baseline by 10,30 and 100 , respectively. For design purposes, the limits can be assumed to range between 0,80 and 1,50 times the recommended values depending on the duration of the vibration and its frequency.

The dynamic analysis of vibrations due to walking is performed according a time dependent harmonic force component that matches the fundamental frequency of the floor, according to the Equation [1].

$F_{i}=P \alpha_{i} \cos \left(2 \pi i f_{\text {step }} t\right)$

where

$\mathrm{P}=$ person's weight, taken as $0,7 \mathrm{kN}$ for design.

$\mathrm{i}=$ harmonic multiple of the step frequency.

$\mathrm{f}_{\text {step }}=$ step frequency. 
$\alpha_{i}=$ dynamic coefficient for the $i^{\text {th }}$ harmonic force component. Recommended values are given in Table [1], however, only a single harmonic is considered at a time since the others would be negligible in comparison to the resonant harmonic.

As such, the resonant response function is given by the Equation [2].

$\frac{a}{g}=\frac{R \alpha_{i} P}{\beta W} \cos \left(2 \pi i f_{\text {step }} t\right)$

in which:

$\frac{a}{g}=$ ratio of the floor acceleration to the acceleration of gravity.

$\beta=$ modal damping ratio.

$W=$ effective weight.

$\mathrm{R}=$ reduction factor that accounts for the fact that full steady-state resonant motion is not achieved for walking and that the excitation source and the affected user are not simultaneously at the location of maximum modal displacement. Taken as 0,7 for footbridges and 0,5 for floor structures with two-way mode shape configurations.

For frequencies above $8 \mathrm{~Hz}$, the quasi-static deflection and footstep impulse vibration can become more critical than resonance. To account for these effects, the AISC recommends that the acceleration should be limited to the same values as the frequencies ranging from $4,0 \mathrm{~Hz}$ to $8,0 \mathrm{~Hz}$ and a minimum stiffness of $1 \mathrm{kN} /$ $\mathrm{mm}$ under concentrated loads should be respected for vibrations of frequencies greater than $10,0 \mathrm{~Hz}$. These criteria, however, are not valid in case equipment sensitive to vibrations are affected.

When considering rhythmic excitations, the design criteria for structures is based on the dynamic response of the structure under loads distributed over the whole floor. They may be used in the evaluation of structural systems submitted to activities such as gymnastics, aerobic and dance classes on the condition that the loading function is known. The peak acceleration of the floor due to harmonic excitations is obtained from the Equation [3] through the classic solution by assuming that the structure presents a single mode of vibration.

$$
\frac{a_{p}}{g}=\frac{1,3 \alpha_{i}{ }^{w_{p}} / w_{t}}{\sqrt{\left[\left(\frac{f_{n}}{f}\right)^{2}-1\right]^{2}+\left[\frac{2 \beta f_{n}}{f}\right]^{2}}}
$$

where:

$\alpha_{i}=$ dynamic coefficient (see Table [1]) $\mathrm{w}_{\mathrm{p}}=$ effective weight per unit area of participants distributed over floor panel.

$\mathrm{w}_{\mathrm{t}}=$ effective weight per unit area of floor panel, including users.

$\mathrm{f}_{\mathrm{n}}=$ natural frequency of the floor $(\mathrm{Hz})$.

$\mathrm{f}=$ excitation frequency $(\mathrm{Hz})$.

$\beta=$ damping ratio.

The effective maximum acceleration accounting for all the harmonic can, then, be calculated from the Equation [4].

$a_{m}=\left[\sum a_{i}^{1,5}\right]^{0,667}$

In which $a_{i}$ represents the peak acceleration for the $i^{\text {th }}$ harmonic.

The damping associated to floor panels is originated from the nonstructural components, furniture and occupants. Damping ratios recommended by the Design Guide range from 0,01 to 0,06 . For footbridges or locations with low occupancy with no furniture nor non-structural components, the damping ratio should be considered 0,01 ; in case of floors with few non-structural components and furniture such as shopping centers and churches the recommended value is 0,02 . A damping ratio of 0,03 is adequate for floors with nonstructural components and furnishings, but with only small demountable partitions, such as modular offices; while a damping ratio of 0,05 should be considered for floors and homes with full height fixed partitions; and a ratio of 0,06 is recommended for vibrations caused by rhythmic excitation due to a great accumulation of people since the users themselves contribute to the damping.

Another important factor to the dynamic analysis of a slab is its own distributed weight, that must be carefully estimated in its actual value for both the live and dead loads, not the design loads. According to the AISC, the live loads should be taken as $0,50 \mathrm{kN} / \mathrm{m}^{2}$ for offices and $0,25 \mathrm{kN} / \mathrm{m}^{2}$ for homes, while footbridges, gymnasiums and shopping centers the live load should be taken as $0 \mathrm{kN} / \mathrm{m}^{2}$.

The application of these criteria, however, demands careful consideration from the engineer. For instance, the acceleration limit for outdoor footbridges is meant for high traffic of people, not quiet places such as office atria.

\section{Methodology}

\subsection{Definition of the standard TMD characteristics}

The basic elements of the proposed TMD can be ascertained from the previously mentioned criteria, and from among the possibilities some

\section{Table 1}

Common excitation frequencies and their respective dynamic coefficients (AISC Steel Design Guides Series 11)

\begin{tabular}{|c|c|c|c|c|c|c|}
\hline \multirow{2}{*}{ Harmonic $\mathbf{i}$} & \multicolumn{2}{|c|}{ Person walking } & \multicolumn{2}{c|}{ Aerobic class } & \multicolumn{2}{c|}{ Group dancing } \\
\cline { 2 - 7 } & $\mathbf{f}, \mathrm{Hz}$ & $\alpha \mathbf{i}$ & $\mathbf{f}, \mathrm{Hz}$ & $\alpha \mathbf{i}$ & $\mathbf{f}, \mathrm{Hz}$ & $\alpha \mathbf{i}$ \\
\hline 1 & $1,60-2,20$ & 0,50 & $2,00-2,75$ & 1,50 & $1,50-3,00$ & 0,50 \\
\hline 2 & $3,20-4,40$ & 0,20 & $4,00-5,55$ & 0,60 & - & - \\
\hline 3 & $4,80-6,60$ & 0,10 & $6,00-8,25$ & 0,10 & - & - \\
\hline 4 & $6,40-8,80$ & 0,05 & - & - & - & - \\
\hline
\end{tabular}


Table 2

Possible distributed weights considered

\begin{tabular}{|c|c|c|c|c|}
\hline \multirow{2}{*}{ Live load } & \multicolumn{3}{|c|}{ H slab } & \multirow{2}{*}{ Floor covering } \\
\hline & $10,0 \mathrm{~cm}$ & $15,0 \mathrm{~cm}$ & $20,0 \mathrm{~cm}$ & \\
\hline $0 \mathrm{Kg} / \mathrm{m}^{2}$ & $250 \mathrm{~kg} / \mathrm{m}^{2}$ & $375 \mathrm{Kg} / \mathrm{m}^{2}$ & $500 \mathrm{Kg} / \mathrm{m}^{2}$ & $0 \mathrm{Kg} / \mathrm{m}^{2}$ \\
\hline $25 \mathrm{Kg} / \mathrm{m}^{2}$ & $275 \mathrm{Kg} / \mathrm{m}^{2}$ & $400 \mathrm{Kg} / \mathrm{m}^{2}$ & $525 \mathrm{~kg} / \mathrm{m}^{2}$ & $0 \mathrm{Kg} / \mathrm{m}^{2}$ \\
\hline $50 \mathrm{Kg} / \mathrm{m}^{2}$ & $300 \mathrm{Kg} / \mathrm{m}^{2}$ & $425 \mathrm{Kg} / \mathrm{m}^{2}$ & $550 \mathrm{Kg} / \mathrm{m}^{2}$ & $0 \mathrm{Kg} / \mathrm{m}^{2}$ \\
\hline $0 \mathrm{Kg} / \mathrm{m}^{2}$ & $350 \mathrm{Kg} / \mathrm{m}^{2}$ & $475 \mathrm{Kg} / \mathrm{m}^{2}$ & $600 \mathrm{Kg} / \mathrm{m}^{2}$ & $100 \mathrm{Kg} / \mathrm{m}^{2}$ \\
\hline $25 \mathrm{Kg} / \mathrm{m}^{2}$ & $375 \mathrm{Kg} / \mathrm{m}^{2}$ & $500 \mathrm{Kg} / \mathrm{m}^{2}$ & $625 \mathrm{Kg} / \mathrm{m}^{2}$ & $100 \mathrm{Kg} / \mathrm{m}^{2}$ \\
\hline $50 \mathrm{Kg} / \mathrm{m}^{2}$ & $400 \mathrm{Kg} / \mathrm{m}^{2}$ & $525 \mathrm{Kg} / \mathrm{m}^{2}$ & $650 \mathrm{Kg} / \mathrm{m}^{2}$ & $100 \mathrm{Kg} / \mathrm{m}^{2}$ \\
\hline $0 \mathrm{Kg} / \mathrm{m}^{2}$ & $450 \mathrm{Kg} / \mathrm{m}^{2}$ & $575 \mathrm{Kg} / \mathrm{m}^{2}$ & $700 \mathrm{Kg} / \mathrm{m}^{2}$ & $200 \mathrm{Kg} / \mathrm{m}^{2}$ \\
\hline $25 \mathrm{Kg} / \mathrm{m}^{2}$ & $475 \mathrm{Kg} / \mathrm{m}^{2}$ & $600 \mathrm{Kg} / \mathrm{m}^{2}$ & $725 \mathrm{Kg} / \mathrm{m}^{2}$ & $200 \mathrm{~kg} / \mathrm{m}^{2}$ \\
\hline $50 \mathrm{Kg} / \mathrm{m}^{2}$ & $500 \mathrm{Kg} / \mathrm{m}^{2}$ & $625 \mathrm{Kg} / \mathrm{m}^{2}$ & $750 \mathrm{Kg} / \mathrm{m}^{2}$ & $200 \mathrm{Kg} / \mathrm{m}^{2}$ \\
\hline
\end{tabular}

will be chosen as standard in a way that covers the greatest range of cases with the least amount of parts. The first elements to be defined are the standard masses. Assuming that the mass of the Tuned-Mass Dampers is contained exclusively on its inertial element, their mass can be obtained from the distributed weight of the structure common on the contemporary practice and desired spacing between mechanisms. Taking in account slabs of $10,0 \mathrm{~cm}, 15,0 \mathrm{~cm}$ and $20,0 \mathrm{~cm}$ of height, floor coverings of $100,0 \mathrm{~kg} / \mathrm{m}^{2}$ or $200 \mathrm{~kg} / \mathrm{m}^{2}$ and live loads varying from $0,0 \mathrm{~kg} /$ $\mathrm{m}^{2}$ to $50,0 \mathrm{~kg} / \mathrm{m}^{2}$, the possible distributed weights are shown in Table [2]. For each possibility of distributed weight, the adequate element mass can be ascertained from the distribution of the mechanisms through the floor and the ratio $\mu$ between the system and structure weights given in Equation [5].

$\mu=\frac{M_{\text {set of } T M D^{\prime} s}}{M_{\text {structure }}}$

The CEB suggest in its Bulletin D'Information N209, annex D, that $\mu$ should usually range from $1,0 \%$ to $5,0 \%$, while Varela and $\mathrm{Ba}$ tista (2011) recommends it should range between $0,2 \%$ to $1,0 \%$. As such, the inertial elements are ascertained assuming $\mu=1,0 \%$ and spacing of $0,50 \mathrm{~m}$ between mechanisms. From the values thus obtained, shown in Table [3], the options of $0,75 \mathrm{~kg}, 1,00 \mathrm{~kg}, 1,25 \mathrm{~kg}$, $1,50 \mathrm{~kg}$ and $1,75 \mathrm{~kg}$ are chosen as the standard masses of the inertial elements in order to better cover the intended range.

Next, the spring stiffness can be defined. Since the TMD's are damped mass-spring oscillators of a single degree of freedom, their natural frequency obeys Equation [6].

$f_{T M D}=\frac{1}{2 \pi} \sqrt{\frac{k}{m}}$

where:

$\mathrm{f}_{T M D}=$ natural frequency of the Tuned-Mass Damper.

$\mathrm{k}=$ system (spring) stiffness.

$\mathrm{m}=$ mass of the inertial element.

The application of TMDs, however, increases the system mass without an equivalent increase in its stiffness, slightly reducing its natural frequency. To compensate this deviation in a simplified manner, the tuning is done for a rectified frequency through Equation [7].

$f_{\text {tuning }}=\frac{1}{1+\mu} f_{\text {natural }}^{\text {floor }}$

in which:

$f_{\text {tuning }}=$ rectified natural frequency of the system.

$f_{\text {natural }}^{\text {floor }}=$ original natural frequency of the system .

According to the AISC the excitation frequency of excitation loads ranges from $1,60 \mathrm{~Hz}$ to $2,20 \mathrm{~Hz}$ for walking, $2,00 \mathrm{~Hz}$ to $2,75 \mathrm{~Hz}$ for aerobic classes and $1,50 \mathrm{~Hz}$ to $3,00 \mathrm{~Hz}$ for dancing. The necessary

\section{Table 3}

Calculated mass of the inertial elements of the TMD for the distributed weights considerer

\begin{tabular}{|c|c|c|c|c|c|c|c|c|c|c|}
\hline $\begin{array}{c}\text { Distributed } \\
\text { weight }\end{array}$ & $\begin{array}{c}250 \\
\mathrm{~kg} / \mathrm{m}^{2}\end{array}$ & $\begin{array}{c}275 \\
\mathrm{~kg} / \mathrm{m}^{2}\end{array}$ & $\begin{array}{c}300 \\
\mathrm{kf} / \mathrm{m}^{2}\end{array}$ & $\begin{array}{c}350 \\
\mathrm{~kg} / \mathrm{m}^{2}\end{array}$ & $\begin{array}{c}375 \\
\mathrm{~kg} / \mathrm{m}^{2}\end{array}$ & $\begin{array}{c}400 \\
\mathrm{~kg} / \mathrm{m}^{2}\end{array}$ & $\begin{array}{c}425 \\
\mathrm{~kg} / \mathrm{m}^{2}\end{array}$ & $\begin{array}{c}450 \\
\mathrm{~kg} / \mathrm{m}^{2}\end{array}$ & $\begin{array}{c}475 \\
\mathrm{~kg} / \mathrm{m}^{2}\end{array}$ & $\begin{array}{c}500 \\
\mathrm{~kg} / \mathrm{m}^{2}\end{array}$ \\
\hline $\begin{array}{c}\text { M for } \\
\mu=1,0 \%\end{array}$ & $\begin{array}{c}0,625 \\
\mathrm{~kg}\end{array}$ & $\begin{array}{c}0,688 \\
\mathrm{~kg}\end{array}$ & $\begin{array}{c}0,750 \\
\mathrm{~kg}\end{array}$ & $\begin{array}{c}0,875 \\
\mathrm{~kg}\end{array}$ & $\begin{array}{c}0,938 \\
\mathrm{~kg}\end{array}$ & $\begin{array}{c}1,000 \\
\mathrm{~kg}\end{array}$ & $\begin{array}{c}1,063 \\
\mathrm{~kg}\end{array}$ & $\begin{array}{c}1,125 \\
\mathrm{~kg}\end{array}$ & $\begin{array}{c}1,188 \\
\mathrm{~kg}\end{array}$ & $\begin{array}{c}1,250 \\
\mathrm{~kg}\end{array}$ \\
\hline $\begin{array}{c}\text { Distributed } \\
\text { weight }\end{array}$ & $\begin{array}{r}525 \\
\mathrm{~kg} / \mathrm{m}^{2}\end{array}$ & $\begin{array}{c}550 \\
\mathrm{~kg} / \mathrm{m}^{2}\end{array}$ & $\begin{array}{c}575 \\
\mathrm{~kg} / \mathrm{m}^{2}\end{array}$ & $\begin{array}{c}600 \\
\mathrm{~kg} / \mathrm{m}^{2}\end{array}$ & $\begin{array}{c}625 \\
\mathrm{~kg} / \mathrm{m}^{2}\end{array}$ & $\begin{array}{c}650 \\
\mathrm{~kg} / \mathrm{m}^{2}\end{array}$ & $\begin{array}{c}700 \\
\mathrm{~kg} / \mathrm{m}^{2}\end{array}$ & $\begin{array}{c}725 \\
\mathrm{~kg} / \mathrm{m}^{2}\end{array}$ & $\begin{array}{c}750 \\
\mathrm{~kg} / \mathrm{m}^{2}\end{array}$ & - \\
\hline $\begin{array}{c}\mathrm{M} \mathrm{for} \\
\mu=1,0 \%\end{array}$ & $\begin{array}{c}1,313 \\
\mathrm{~kg}\end{array}$ & $\begin{array}{c}1,375 \\
\mathrm{~kg}\end{array}$ & $\begin{array}{c}1,438 \\
\mathrm{~kg}\end{array}$ & $\begin{array}{c}1,500 \\
\mathrm{~kg}\end{array}$ & $\begin{array}{c}1,563 \\
\mathrm{~kg}\end{array}$ & $\begin{array}{c}1,625 \\
\mathrm{~kg}\end{array}$ & $\begin{array}{c}1,750 \\
\mathrm{~kg}\end{array}$ & $\begin{array}{c}1,813 \\
\mathrm{~kg}\end{array}$ & $\begin{array}{c}1,875 \\
\mathrm{~kg}\end{array}$ & - \\
\hline
\end{tabular}




\section{Table 4}

Calculated stiffness of the springs of the TMD based on the inertial element and frequencies selected

\begin{tabular}{|c|c|c|c|}
\hline \multirow{2}{*}{$\mathbf{f}$} & \multicolumn{2}{|c|}{ M } & $\mathbf{1}, \mathbf{7 5 0 K g}$ \\
\cline { 2 - 4 } & $\mathbf{0 , 7 5 0 K g}$ & $1,250 \mathrm{Kg}$ & $152,38 \mathrm{~N} / \mathrm{m}$ \\
\hline $1,50 \mathrm{~Hz}$ & $65,31 \mathrm{~N} / \mathrm{m}$ & $193,50 \mathrm{~N} / \mathrm{m} / \mathrm{m}$ & $270,90 \mathrm{~N} / \mathrm{m}$ \\
\hline $2,00 \mathrm{~Hz}$ & $116,10 \mathrm{~N} / \mathrm{m}$ & $302,35 \mathrm{~N} / \mathrm{m}$ & $609,53 \mathrm{~N} / \mathrm{m}$ \\
\hline $2,50 \mathrm{~Hz}$ & $181,41 \mathrm{~N} / \mathrm{m}$ & $435,38 \mathrm{~N} / \mathrm{m}$ & $829,64 \mathrm{~N} / \mathrm{m}$ \\
\hline $3,00 \mathrm{~Hz}$ & $261,23 \mathrm{~N} / \mathrm{m}$ & $592,60 \mathrm{~N} / \mathrm{m}$ & $1083,62 \mathrm{~N} / \mathrm{m}$ \\
\hline $3,50 \mathrm{~Hz}$ & $355,56 \mathrm{~N} / \mathrm{m}$ & $774,01 \mathrm{~N} / \mathrm{m}$ & $1371,45 \mathrm{~N} / \mathrm{m}$ \\
\hline $4,00 \mathrm{~Hz}$ & $464,41 \mathrm{~N} / \mathrm{m}$ & $979,61 \mathrm{~N} / \mathrm{m}$ & $1693,15 \mathrm{~N} / \mathrm{m}$ \\
\hline $4,50 \mathrm{~Hz}$ & $587,76 \mathrm{~N} / \mathrm{m}$ & $1209,39 \mathrm{~N} / \mathrm{m}$ & $2048,71 \mathrm{~N} / \mathrm{m}$ \\
\hline $5,00 \mathrm{~Hz}$ & $725,64 \mathrm{~N} / \mathrm{m}$ & $1463,36 \mathrm{~N} / \mathrm{m}$ & $2438,13 \mathrm{~N} / \mathrm{m}$ \\
\hline $5,50 \mathrm{~Hz}$ & $878,02 \mathrm{~N} / \mathrm{m}$ & $1741,52 \mathrm{~N} / \mathrm{m}$ & $2861,42 \mathrm{~N} / \mathrm{m}$ \\
\hline $6,00 \mathrm{~Hz}$ & $1044,91 \mathrm{~N} / \mathrm{m}$ & $2043,87 \mathrm{~N} / \mathrm{m}$ & $3318,57 \mathrm{~N} / \mathrm{m}$ \\
\hline $6,50 \mathrm{~Hz}$ & $1226,32 \mathrm{~N} / \mathrm{m}$ & $2370,41 \mathrm{~N} / \mathrm{m}$ & $3809,58 \mathrm{~N} / \mathrm{m}$ \\
\hline $7,00 \mathrm{~Hz}$ & $1422,24 \mathrm{~N} / \mathrm{m}$ & $2721,13 \mathrm{~N} / \mathrm{m}$ & $4334,46 \mathrm{~N} / \mathrm{m}$ \\
\hline $7,50 \mathrm{~Hz}$ & $1632,68 \mathrm{~N} / \mathrm{m}$ & $3096,04 \mathrm{~N} / \mathrm{m}$ & \\
\hline $8,00 \mathrm{~Hz}$ & $1857,63 \mathrm{~N} / \mathrm{m}$ & & \\
\hline
\end{tabular}

springs to comprise the whole range of excitation and their harmonics can be obtained using Equations [6] and [7], and are indicate in Table [4]. Among the resulting stiffness, fifteen are selected as standard: $100 \mathrm{~N} / \mathrm{m}, 150 \mathrm{~N} / \mathrm{m}, 225 \mathrm{~N} / \mathrm{m}, 330 \mathrm{~N} / \mathrm{m}, 500 \mathrm{~N} / \mathrm{m}, 630 \mathrm{~N} / \mathrm{m}$, $790 \mathrm{~N} / \mathrm{m}, 1000 \mathrm{~N} / \mathrm{m}, 1235 \mathrm{~N} / \mathrm{m}, 1500 \mathrm{~N} / \mathrm{m}, 1780 \mathrm{~N} / \mathrm{m}, 2050 \mathrm{~N} / \mathrm{m}$,
$2350 \mathrm{~N} / \mathrm{m}, 2700 \mathrm{~N} / \mathrm{m}$ e $3100 \mathrm{~N} / \mathrm{m}$; that, together with the standard masses previously selected, allow the tuning of the mechanism to the exact frequencies indicated in Table [5].

Lastly, the standard dampers must be defined. According to the Bulletin D'Information N209, annex D, the optimum damping ratio

\section{Table 5}

Possible rectified natural frequencies for the mass-spring combinations of the standard TMD

\begin{tabular}{|c|c|c|c|c|c|}
\hline \multirow{2}{*}{ k } & \multicolumn{5}{|c|}{ M } \\
\hline & $0,750 \mathrm{Kg}$ & $1,000 \mathrm{Kg}$ & $1,250 \mathrm{Kg}$ & $1,500 \mathrm{Kg}$ & $1,750 \mathrm{Kg}$ \\
\hline $100 \mathrm{~N} / \mathrm{m}$ & $1,86 \mathrm{~Hz}$ & $1,61 \mathrm{~Hz}$ & $1,44 \mathrm{~Hz}$ & $1,31 \mathrm{~Hz}$ & 1,22 Hz \\
\hline $150 \mathrm{~N} / \mathrm{m}$ & $2,27 \mathrm{~Hz}$ & 1,97 Hz & 1,76 Hz & 1,61 Hz & 1,49 Hz \\
\hline $225 \mathrm{~N} / \mathrm{m}$ & $2,78 \mathrm{~Hz}$ & $2,41 \mathrm{~Hz}$ & $2,16 \mathrm{~Hz}$ & 1,97 Hz & $1,82 \mathrm{~Hz}$ \\
\hline $330 \mathrm{~N} / \mathrm{m}$ & $3,37 \mathrm{~Hz}$ & $2,92 \mathrm{~Hz}$ & $2,61 \mathrm{~Hz}$ & $2,38 \mathrm{~Hz}$ & $2,21 \mathrm{~Hz}$ \\
\hline $500 \mathrm{~N} / \mathrm{m}$ & $4,15 \mathrm{~Hz}$ & $3,59 \mathrm{~Hz}$ & $3,21 \mathrm{~Hz}$ & $2,93 \mathrm{~Hz}$ & $2,72 \mathrm{~Hz}$ \\
\hline $630 \mathrm{~N} / \mathrm{m}$ & $4,66 \mathrm{~Hz}$ & 4,03 Hz & $3,61 \mathrm{~Hz}$ & $3,29 \mathrm{~Hz}$ & $3,05 \mathrm{~Hz}$ \\
\hline 790 N/m & $5,22 \mathrm{~Hz}$ & $4,52 \mathrm{~Hz}$ & $4,04 \mathrm{~Hz}$ & $3,69 \mathrm{~Hz}$ & $3,42 \mathrm{~Hz}$ \\
\hline 1000 N/m & $5,87 \mathrm{~Hz}$ & $5,08 \mathrm{~Hz}$ & $4,55 \mathrm{~Hz}$ & $4,15 \mathrm{~Hz}$ & $3,84 \mathrm{~Hz}$ \\
\hline $1235 \mathrm{~N} / \mathrm{m}$ & $6,52 \mathrm{~Hz}$ & $5,65 \mathrm{~Hz}$ & $5,05 \mathrm{~Hz}$ & $4,61 \mathrm{~Hz}$ & $4,27 \mathrm{~Hz}$ \\
\hline $1500 \mathrm{~N} / \mathrm{m}$ & $7,19 \mathrm{~Hz}$ & $6,23 \mathrm{~Hz}$ & $5,57 \mathrm{~Hz}$ & $5,08 \mathrm{~Hz}$ & $4,71 \mathrm{~Hz}$ \\
\hline 1780 N/m & $7,83 \mathrm{~Hz}$ & $6,78 \mathrm{~Hz}$ & $6,07 \mathrm{~Hz}$ & $5,54 \mathrm{~Hz}$ & $5,13 \mathrm{~Hz}$ \\
\hline 2050 N/m & $8,40 \mathrm{~Hz}$ & $7,28 \mathrm{~Hz}$ & $6,51 \mathrm{~Hz}$ & $5,94 \mathrm{~Hz}$ & $5,50 \mathrm{~Hz}$ \\
\hline $2350 \mathrm{~N} / \mathrm{m}$ & $9,00 \mathrm{~Hz}$ & 7,79 Hz & $6,97 \mathrm{~Hz}$ & $6,36 \mathrm{~Hz}$ & $5,89 \mathrm{~Hz}$ \\
\hline $2700 \mathrm{~N} / \mathrm{m}$ & $9,64 \mathrm{~Hz}$ & $8,35 \mathrm{~Hz}$ & $7,47 \mathrm{~Hz}$ & $6,82 \mathrm{~Hz}$ & $6,31 \mathrm{~Hz}$ \\
\hline $3100 \mathrm{~N} / \mathrm{m}$ & $10,33 \mathrm{~Hz}$ & $8,95 \mathrm{~Hz}$ & $8,01 \mathrm{~Hz}$ & 7,31 Hz & $6,77 \mathrm{~Hz}$ \\
\hline
\end{tabular}


Table 6

Ideal damping coefficient for the considered spring-frequency combinations

\begin{tabular}{|c|c|c|c|c|c|c|}
\hline \multirow{2}{*}{$\omega$} & \multicolumn{5}{|c|}{$\mathbf{M}$} & $\mathbf{1}$ \\
\cline { 2 - 7 } & $\mathbf{0 , 7 5 0} \mathbf{K g}$ & $\mathbf{1 , 0 0 0} \mathbf{K g}$ & $\mathbf{1 , 2 5 0} \mathbf{K g}$ & $\mathbf{1 , 5 0 0 K g}$ & $\mathbf{f}$ \\
\hline $9,42 \mathrm{rad} / \mathrm{s}$ & $0,85 \mathrm{Ns} / \mathrm{m}$ & $1,14 \mathrm{Ns} / \mathrm{m}$ & $1,42 \mathrm{Ns} / \mathrm{m}$ & $1,71 \mathrm{Ns} / \mathrm{m}$ & $1,99 \mathrm{Ns} / \mathrm{m}$ & $1,50 \mathrm{~Hz}$ \\
\hline $12,57 \mathrm{rad} / \mathrm{s}$ & $1,14 \mathrm{Ns} / \mathrm{m}$ & $1,52 \mathrm{Ns} / \mathrm{m}$ & $1,90 \mathrm{Ns} / \mathrm{m}$ & $2,27 \mathrm{Ns} / \mathrm{m}$ & $2,65 \mathrm{Ns} / \mathrm{m}$ & $2,00 \mathrm{~Hz}$ \\
\hline $15,71 \mathrm{rad} / \mathrm{s}$ & $1,42 \mathrm{Ns} / \mathrm{m}$ & $1,90 \mathrm{Ns} / \mathrm{m}$ & $2,37 \mathrm{Ns} / \mathrm{m}$ & $2,84 \mathrm{Ns} / \mathrm{m}$ & $3,32 \mathrm{Ns} / \mathrm{m}$ & $2,50 \mathrm{~Hz}$ \\
\hline $18,85 \mathrm{rad} / \mathrm{s}$ & $1,71 \mathrm{Ns} / \mathrm{m}$ & $2,27 \mathrm{Ns} / \mathrm{m}$ & $2,84 \mathrm{Ns} / \mathrm{m}$ & $3,41 \mathrm{Ns} / \mathrm{m}$ & $3,98 \mathrm{Ns} / \mathrm{m}$ & $3,00 \mathrm{~Hz}$ \\
\hline $21,99 \mathrm{rad} / \mathrm{s}$ & $1,99 \mathrm{Ns} / \mathrm{m}$ & $2,65 \mathrm{Ns} / \mathrm{m}$ & $3,32 \mathrm{Ns} / \mathrm{m}$ & $3,98 \mathrm{Ns} / \mathrm{m}$ & $4,64 \mathrm{Ns} / \mathrm{m}$ & $3,50 \mathrm{~Hz}$ \\
\hline $25,13 \mathrm{rad} / \mathrm{s}$ & $2,27 \mathrm{Ns} / \mathrm{m}$ & $3,03 \mathrm{Ns} / \mathrm{m}$ & $3,79 \mathrm{Ns} / \mathrm{m}$ & $4,55 \mathrm{Ns} / \mathrm{m}$ & $5,31 \mathrm{Ns} / \mathrm{m}$ & $4,00 \mathrm{~Hz}$ \\
\hline $28,27 \mathrm{rad} / \mathrm{s}$ & $2,56 \mathrm{Ns} / \mathrm{m}$ & $3,41 \mathrm{Ns} / \mathrm{m}$ & $4,26 \mathrm{Ns} / \mathrm{m}$ & $5,12 \mathrm{Ns} / \mathrm{m}$ & $5,97 \mathrm{Ns} / \mathrm{m}$ & $4,50 \mathrm{~Hz}$ \\
\hline $31,42 \mathrm{rad} / \mathrm{s}$ & $2,84 \mathrm{Ns} / \mathrm{m}$ & $3,79 \mathrm{Ns} / \mathrm{m}$ & $4,74 \mathrm{Ns} / \mathrm{m}$ & $5,69 \mathrm{Ns} / \mathrm{m}$ & $6,63 \mathrm{Ns} / \mathrm{m}$ & $5,00 \mathrm{~Hz}$ \\
\hline $34,56 \mathrm{rad} / \mathrm{s}$ & $3,13 \mathrm{Ns} / \mathrm{m}$ & $4,17 \mathrm{Ns} / \mathrm{m}$ & $5,21 \mathrm{Ns} / \mathrm{m}$ & $6,25 \mathrm{Ns} / \mathrm{m}$ & $7,30 \mathrm{Ns} / \mathrm{m}$ & $5,50 \mathrm{~Hz}$ \\
\hline $37,70 \mathrm{rad} / \mathrm{s}$ & $3,41 \mathrm{Ns} / \mathrm{m}$ & $4,55 \mathrm{Ns} / \mathrm{m}$ & $5,69 \mathrm{Ns} / \mathrm{m}$ & $6,82 \mathrm{Ns} / \mathrm{m}$ & $7,96 \mathrm{Ns} / \mathrm{m}$ & $6,00 \mathrm{~Hz}$ \\
\hline $43,98 \mathrm{rad} / \mathrm{s}$ & $3,98 \mathrm{Ns} / \mathrm{m}$ & $5,31 \mathrm{Ns} / \mathrm{m}$ & $6,63 \mathrm{Ns} / \mathrm{m}$ & $7,96 \mathrm{Ns} / \mathrm{m}$ & $9,29 \mathrm{Ns} / \mathrm{m}$ & $7,00 \mathrm{~Hz}$ \\
\hline $47,12 \mathrm{rad} / \mathrm{s}$ & $4,26 \mathrm{Ns} / \mathrm{m}$ & $5,69 \mathrm{Ns} / \mathrm{m}$ & $7,11 \mathrm{Ns} / \mathrm{m}$ & $8,53 \mathrm{Ns} / \mathrm{m}$ & $9,95 \mathrm{Ns} / \mathrm{m}$ & $7,50 \mathrm{~Hz}$ \\
\hline $50,27 \mathrm{rad} / \mathrm{s}$ & $4,55 \mathrm{Ns} / \mathrm{m}$ & $6,07 \mathrm{Ns} / \mathrm{m}$ & $7,58 \mathrm{Ns} / \mathrm{m}$ & $9,10 \mathrm{Ns} / \mathrm{m}$ & $10,61 \mathrm{Ns} / \mathrm{m}$ & $8,00 \mathrm{~Hz}$ \\
\hline
\end{tabular}

of a Tuned-Mass Damper can be obtained in a simplified manner from the Equation [8].

$\xi_{\text {optimum }}=\left[\frac{3 \mu}{8(1+\mu)^{3}}\right]^{1 / 2}$

where $\xi$ is calculated from Equation [9].

$\xi=\frac{c}{2 m \varpi}$

in which:

$\mathrm{c}=$ the damping coefficient.

$\mathrm{m}=$ mass of the inertial element.

$\omega=$ natural angular frequency of the TMD.

The ideal damping coefficient for each mass and frequency combination are given in Table [6]. Among those, $1,00 \mathrm{Ns} / \mathrm{m}, 1,75 \mathrm{Ns} / \mathrm{m}$, $2,50 \mathrm{Ns} / \mathrm{m}, 3,25 \mathrm{Ns} / \mathrm{m}, 4,00 \mathrm{Ns} / \mathrm{m}, 4,60 \mathrm{Ns} / \mathrm{m}, 5,20 \mathrm{Ns} / \mathrm{m}, 6,00 \mathrm{Ns} / \mathrm{m}$ $7,00 \mathrm{Ns} / \mathrm{m}$ e $8,00 \mathrm{Ns} / \mathrm{m}$ were chosen as standard to better comprise the range of interest.

In short, the Tuned-Mass Damper to be used can be applied basically to any case within the analyzed spectrum through the compounding of the mechanism from five possible weights, fifteen springs and ten dampers, respectively indicated in Tables [7], [8] and [9].

\subsection{Guide for parts selection of the tuned-mass damper}

After recognizing the vibration problems on the floor, the assemblage of the TMD is straightforward and simples. Initially, the equivalent distributed weight is ascertained, in $\mathrm{kg} / \mathrm{m}^{2}$, from the sum of the weight of the slab, floor covering and live load in their actual values. The ideal inertial element for the mechanism can, thus, be calculated by multiplying this value by $\mu=1,00 \%$ and the influence area of each TMD, that is, the area obtained from the spacing of each adjacent mechanism, as demonstrated in Equation [10].

Table 7

Mass of the standard inertial elements (weights)

\begin{tabular}{|c|c|c|c|c|}
\hline \multicolumn{5}{|c|}{$M$} \\
\hline $0,750 \mathrm{Kg}$ & $1,000 \mathrm{Kg}$ & $1,250 \mathrm{Kg}$ & $1,500 \mathrm{Kg}$ & $1,750 \mathrm{Kg}$ \\
\hline
\end{tabular}

Table 8

Stiffness of the standard restorative elements (springs)

\begin{tabular}{|c|c|c|c|c|}
\hline \multicolumn{5}{|c|}{$M$} \\
\hline $100 \mathrm{~N} / \mathrm{m}$ & $150 \mathrm{~N} / \mathrm{m}$ & $225 \mathrm{~N} / \mathrm{m}$ & $330 \mathrm{~N} / \mathrm{m}$ & $500 \mathrm{~N} / \mathrm{m}$ \\
\hline $630 \mathrm{~N} / \mathrm{m}$ & $790 \mathrm{~N} / \mathrm{m}$ & $1000 \mathrm{~N} / \mathrm{m}$ & $1235 \mathrm{~N} / \mathrm{m}$ & $1500 \mathrm{~N} / \mathrm{m}$ \\
\hline $1780 \mathrm{~N} / \mathrm{m}$ & $2050 \mathrm{~N} / \mathrm{m}$ & $2350 \mathrm{~N} / \mathrm{m}$ & $2700 \mathrm{~N} / \mathrm{m}$ & $3100 \mathrm{~N} / \mathrm{m}$ \\
\hline
\end{tabular}


Table 9

Damping coefficient of the standard dissipative elements (dampers)

\begin{tabular}{|c|l|l|l|l|}
\hline \multicolumn{5}{|c|}{ C } \\
\hline $1,00 \mathrm{Ns} / \mathrm{m}$ & $1,75 \mathrm{Ns} / \mathrm{m}$ & $2,50 \mathrm{Ns} / \mathrm{m}$ & $3,25 \mathrm{Ns} / \mathrm{m}$ & $4,00 \mathrm{Ns} / \mathrm{m}$ \\
\hline $4,60 \mathrm{Ns} / \mathrm{m}$ & $5,20 \mathrm{Ns} / \mathrm{m}$ & $6,00 \mathrm{Ns} / \mathrm{m}$ & $7,00 \mathrm{Ns} / \mathrm{m}$ & $8,00 \mathrm{Ns} / \mathrm{m}$ \\
\hline
\end{tabular}

$M_{T M D}^{\text {calculated }}=$

$\mu\left[\left(h_{\text {slab }} \rho_{\text {concrete }}+h_{f . \text { covering }} \rho_{\text {f. covering }}+Q_{\text {AISC }}\right) d_{x} d_{y}\right]$

na qual:

$\mu$ is taken as $1,00 \%$;

$\mathrm{h}_{\text {slab }}=$ slab height $(\mathrm{m})$;

$\mathrm{h}_{\mathrm{f} \text {. covering }}=$ floor covering height $(\mathrm{m})$;

$\rho_{\text {concrete }}=$ reinforced concrete density $\left(\mathrm{kg} / \mathrm{m}^{3}\right)$;

pf. covering = density of the floor covering $\left(\mathrm{kg} / \mathrm{m}^{3}\right)$;

QAISC = equivalent weight of the live loads used for dynamic analysis (recommended by the AISC Design Guide 11 as approximately $50 \mathrm{~kg} / \mathrm{m}^{2}$ for offices, $25 \mathrm{~kg} / \mathrm{m}^{2}$ for houses and $0 \mathrm{~kg} / \mathrm{m}^{2}$ for footbridges, gymnasiums and shopping centers);

$\mathrm{d}_{\mathrm{x}}=$ spacing between TMD's in the main direction of the slab $(\mathrm{m})$; $d_{y}=$ spacing between TMD's in the secondary direction of the slab (m). The inertial element is then chosen as the one closest to the calculated value among the options in Table [7]. The next step would then be choosing the spring stiffness, which will be ascertained from the chosen inertial element and the natural frequency of the structure that needs mitigating, as seen in Equation [11], obtained from the Equations [6] and [7]. The resulting stiffness is then used as basis for choosing the spring among the options in Table [8].

$k_{T M D}^{\text {Calculated }}=\left[\frac{2 \pi}{(1+\mu)} f_{\text {natural }}^{\text {floor }}\right]^{2} \times M_{T M D}^{\text {Adopted }}$
Lastly, the ideal damping is calculated according to Equation 12 that results from manipulating the Equations [8] and [9]. The damper can then be chosen from the options indicated in Table [9].

$c_{T M D}^{\text {Caldulated }}=\left[\frac{3 \mu \times M_{T M D}^{\text {Adopted }} \times k_{T M D}^{\text {Adopted }}}{2(1+\mu)^{3}}\right]^{1 / 2}$

\subsection{Case analysis}

TTo verify the efficacy of the TMD assembled according the process described in the previous item, a case analysis was performed on an office floor made of concrete, $f_{c k} 40 \mathrm{Mpa}$; height of $10,0 \mathrm{~cm}$; spans of $580,0 \mathrm{~cm}$ by $600,0 \mathrm{~cm} ; 2,0 \mathrm{~cm}$ concrete covering thickness and subjected to the following loads: self-weight of $2500 \mathrm{~N} / \mathrm{m}^{2}$, floor covering of $2000 \mathrm{~N} / \mathrm{m}^{2}$ and live loads of $2000 \mathrm{~N} / \mathrm{m}^{2}$. The resulting moments, obtained using the Tables of Czerny for slabs with four simply supported sides, are $m_{x s d}=10220 \mathrm{Nm} / \mathrm{m}$ e $m_{\text {ysd }}=9690 \mathrm{Nm} / \mathrm{m}$. Considering CA-50 steel and respecting requirements from the ABNT NBR 6118:2013, the necessary reinforcement of the slabs is $4,65 \mathrm{~cm}^{2} / \mathrm{m}$ on the main direction and $5,18 \mathrm{~cm}^{2} / \mathrm{m}$ on the secondary direction, both satisfied by $\Phi 10 \mathrm{c} / 15 \mathrm{~cm}$. The resulting deflection is $19,5 \mathrm{~mm}$, less than the required limit of $1 / 300$ if the main span. Next, assuming the slab is held by four beams $20 \mathrm{~cm}$ wide and $45 \mathrm{~cm}$ tall, with concrete covering thickness of $2,50 \mathrm{~cm}$, the critical beam is submitted to $6700 \mathrm{~N} / \mathrm{m}$ dead load, $3000 \mathrm{~N} / \mathrm{m}$ live load and $2250 \mathrm{~N} / \mathrm{m}$ self-weight with a span of $6,00 \mathrm{~m}$. Thus, the

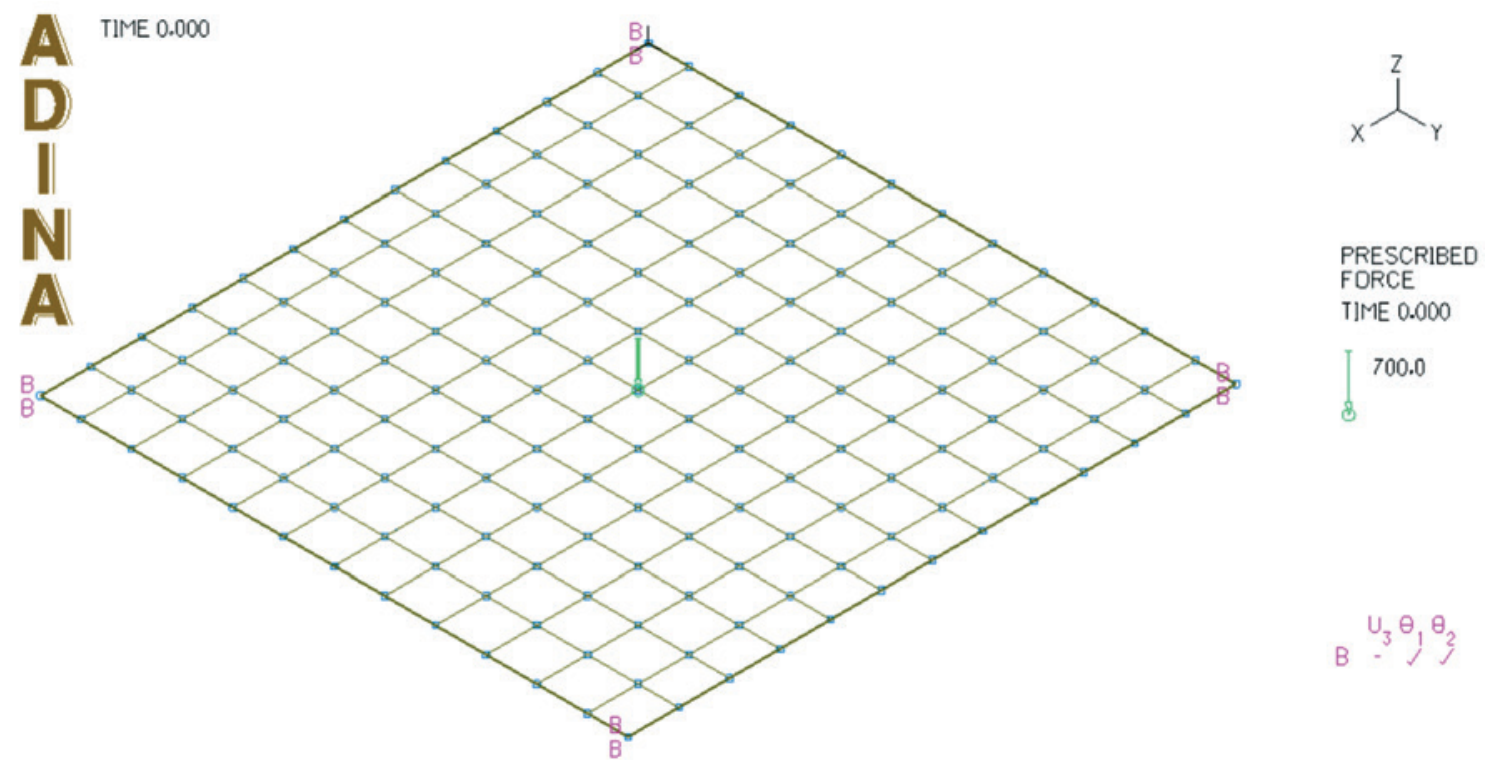

\section{Figure 2}

Modeling of the analyzed floor without TMD's on the Adina 9,1 software 


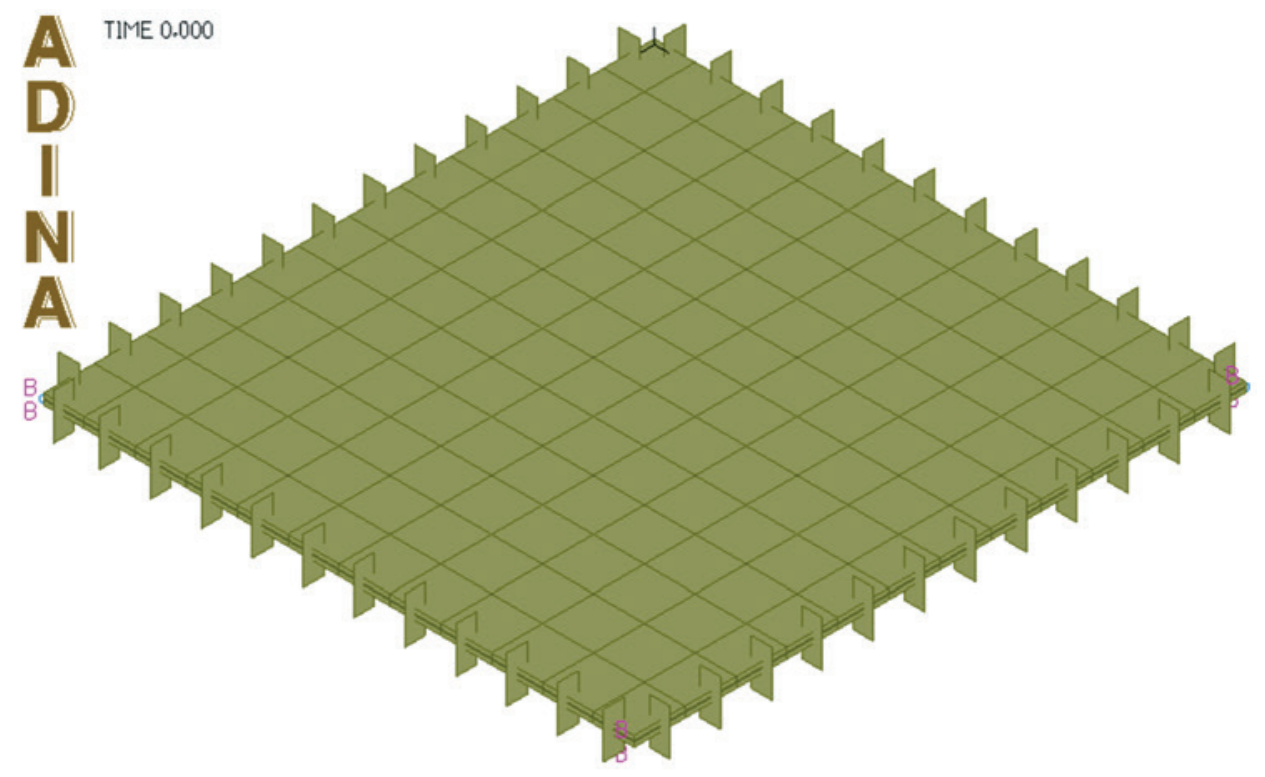

Figure 3

Rendering of the analyzed floor without TMD's on the Adina 9,1 software

resulting moment is $\mathrm{M}_{\mathrm{sd}}=76640 \mathrm{Nm}$, that, neglecting the slab contribution, requires $4,65 \mathrm{~cm}^{2}$ reinforcement., satisfied by the adoption of $4 \Phi 12,5$. The resulting deflection is $1,23 \mathrm{~cm}$ and the maximum fissure gap is $0,08 \mathrm{~mm}$, both values satisfy the requirements of the NBR 6118:2013. In short, the structure is compatible with the ABNT requirements for static design.

To Figure the natural frequencies, amplitude of displacements and accelerations of the structural system before and after the setup of the TMD's, the software Adina 9,1, student license was utilized. A model was created with unities from the International System, a concentrated sinusoidal harmonic load of $700 \mathrm{~N}$ applied to the center of the slab and fixed supports on its vertices. The following Figures [2] and [3] represent respectively the simple and rendered images of the model as seen in the software.

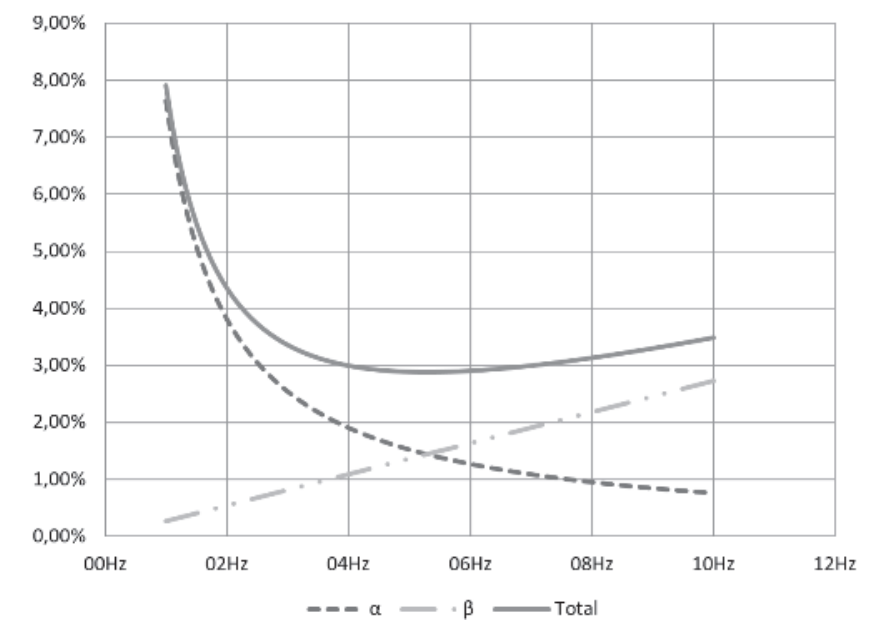

Figure 4

Resulting Rayleigh damping
From the analysis of the model, the following natural frequencies are obtained: $5,62 \mathrm{~Hz}$ for the first mode, $11,56 \mathrm{~Hz}$ for the second mode and $11,86 \mathrm{~Hz}$ for the third mode. Among those, only the first is contained within the range of frequencies generated due to walking and thus the Tuned-Mass Damper will be tuned for $5,62 \mathrm{~Hz}$.

The choice of inertial element is done by applying Equation [10] to the case with a dynamic live load of $50 \mathrm{~kg} / \mathrm{m}^{2}$, that is, $500 \mathrm{~N} / \mathrm{m}^{2}$, recommended by the Design Guide for office floors:

$M_{T M D}^{\text {calculated }}=$

$0,01[(0,10 \times 2500+200+50) 0,50 \times 0,50]=$

$1,25 \mathrm{~kg} \rightarrow M_{T M D}^{\text {adopted }}=1,25 \mathrm{Kg}$

Next, the ideal spring stiffness is ascertained from Equation [11]:

$k_{T M D}^{\text {calculated }}=\left[\frac{2 \pi}{(1+0,01)} 5,62\right]^{2} \times 1,25=1528 \mathrm{~N} / \mathrm{m}$

$\rightarrow k_{T M D}^{\text {adopted }}=1500 \mathrm{~N} / \mathrm{m}$

Lastly, the ideal damping is obtained by applying Equation [12] to the case:

$c_{T M D}^{\text {calculated }}=\left[\frac{3 \times 0,01 \times 1,25 \times 1500}{2(1+0,01)^{3}}\right]^{1 / 2}=5,22 \mathrm{Ns} / \mathrm{m}$

$\rightarrow c_{T M D}^{\text {adopted }}=5,20 \mathrm{Ns} / \mathrm{m}$

A new model is then created with the application of the previously defined Tuned-Mass Dampers under the slab, spaced 0,50m from each other. The natural frequency of this new model is $5,30 \mathrm{~Hz}$ for the first mode.

To accommodate all the AISC demands, the exciting load is multiplied by an $\alpha i$ coefficient, indicated in Table [1] for each harmonic. This means that the maximum intensity of the excitation load varies from one harmonic to the next. Also, according to the AISC recommendation the damping ratio considered is that of offices, 0,03 , and will be considered through the application of Rayleigh 
Damping, in which the damping ratio is obtained from Equation [13] with two variables, $\alpha_{\text {Rayleigh }}$ and $\beta_{\text {Rayleigh }}$, and is defined in such a way that results in a value as close as possible to the desired for the greater part of the analyzed range. To guarantee a damping ratio of 0,03 for excitations close the resonance frequency, the Rayleigh damping will be adjusted to present the desired ratio for $4,00 \mathrm{~Hz}$ and $7,00 \mathrm{~Hz}$, which results in the variables $\alpha_{\text {Rayleigh }}=0,9596$ and $\beta_{\text {Rayleigh }}=8,68 \times 10^{-4}$ and the damping ratio shown in Figure [4]. Then, varying the excitation frequency through the interest range the stabilized displacement and acceleration amplitudes can be obtained. $\xi_{n}=\frac{1}{2 \omega_{n}} \alpha_{\text {Rayleigh }}+\frac{\omega_{n}}{2} \beta_{\text {Rayleigh }}$

\section{Results and discussions}

The difference in response between the structural systems with and without the application of TMD's represented in the previous two models can be visualized in the graphics of the Figures [5] and [6] that present the root mean square values of the acceleration and displacement in the center of the slab for the stabilized

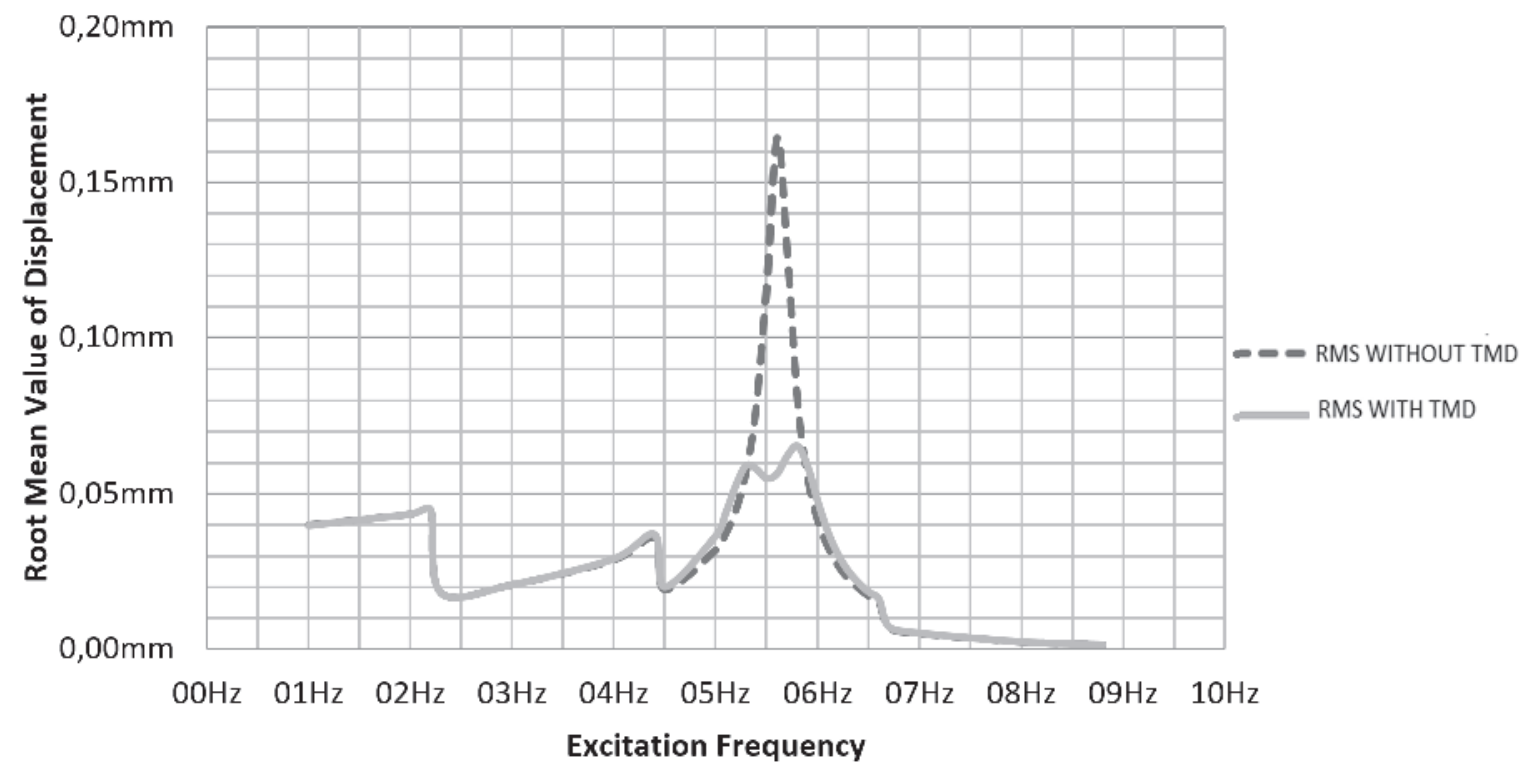

Figure 5

Root mean value of displacement $x$ frequency

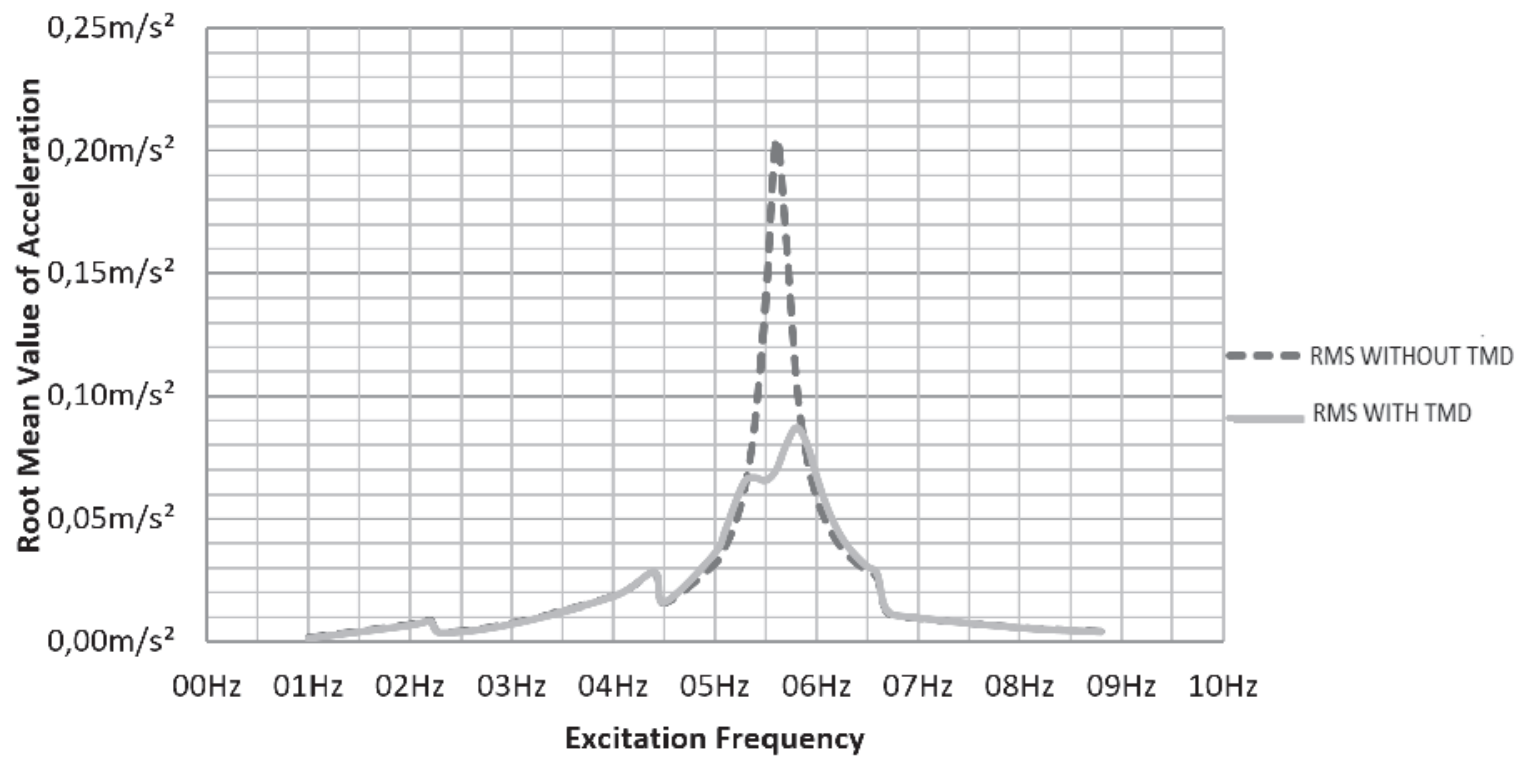

Figure 6

Root mean value of acceleration $x$ frequency 


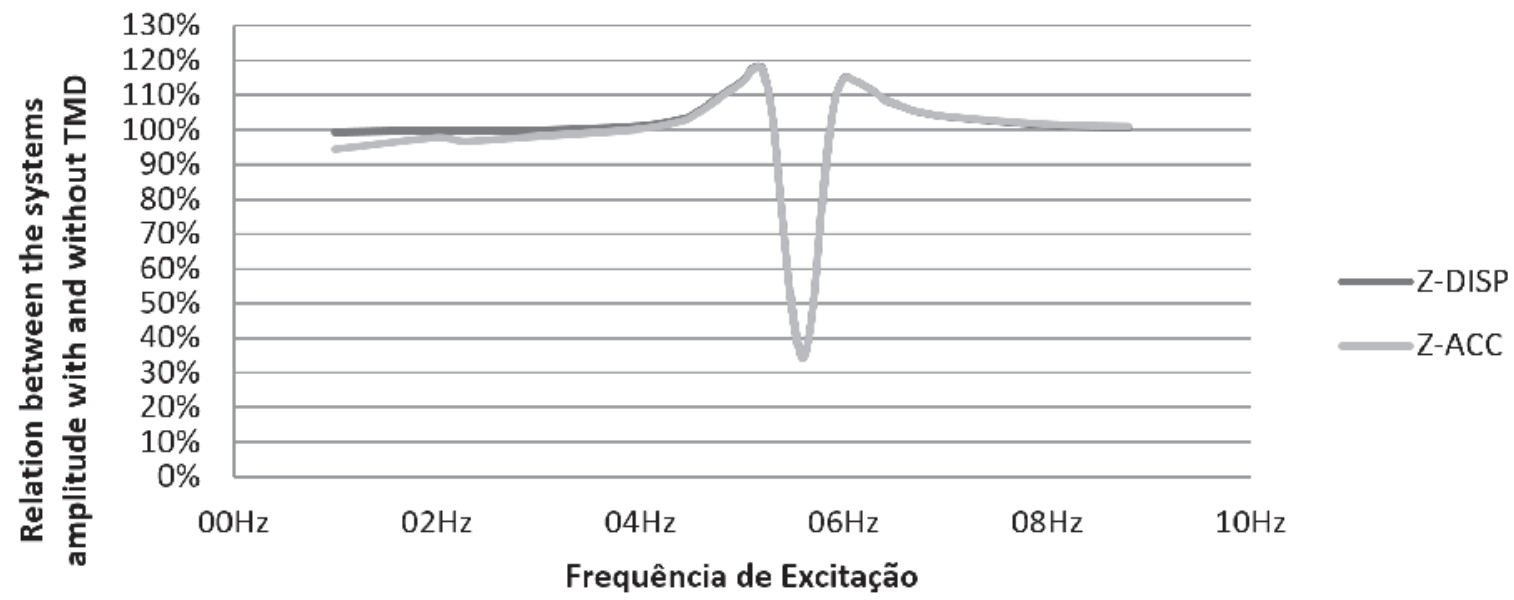

\section{Figure 7}

Relation Between the systems amplitude with and without TMD's

harmonic system respectively. Based in those results, the reduction of the resonant amplitude of displacement and acceleration is clear. The root mean square values of both are reduced close to $65 \%$ for the exciting frequency of $5,6 \mathrm{~Hz}$, the displacement diminishing from $0,165 \mathrm{~mm}$ to $0,057 \mathrm{~mm}$ and the acceleration is reduced from $0,204 \mathrm{~m} / \mathrm{s}^{2}$ to $0,07 \mathrm{~m} / \mathrm{s}^{2}$. It should be noted that even though those results seem negligible in the first place, the displacement is based only on a single concentrated load of $700 \mathrm{~N}$, much lesser than the real loading; and the acceleration with and without TMD's represent $2,06 \%$ and $0,71 \%$ of the gravity respectively, while the acceleration limit for offices should range from $0,40 \%$ and $0,75 \%$. Also, in Figures [5] and [6] the noncontinuities that occur for $2,20 \mathrm{~Hz}, 4,40 \mathrm{~Hz}$ and $6,60 \mathrm{~Hz}$ are caused by the change of the coefficient ai of each harmonic. The difference in behavior of the structural system before and after the setup of the TMD's is clear not only due to the reduction of the dynamic response at the resonance frequency but also due to the emergence of two new dynamic peaks besides the new generated valley, which actually possess worse dynamic response than the original system. The presence of those peaks, however, was already expected as a side effect of the use of Tuned-Mass Dampers and is lessened by the fact that the two new maximums are dwarfed when compared to the original peak. To better represent this behavior, the graphic of Figure [7] indicates the efficacy of the use of the TMD's by directly comparing the results with and without the mechanisms for the whole range of analyzed excitation frequencies.

\section{Conclusion}

This study indicates a reduction of about $65 \%$ of the root mean square values of acceleration, proving the efficiency of the suggest solution. Another advantage of said method are its relative easiness of use and installation. This study, however, was limited to a single case, making it necessary to develop new models that account for a broader range of typical structural engineering situations, with different occupancies, geometric shapes and spans.

\section{Acknowledgements}

This study was possible due to the support of ADINA R\&D. Inc that freely provided the Adina 9,1 software, 900 nodes edition, for students from all over the world

\section{Bibliographic References}

[1] AMERICAN INSTITUTE OF STEEL CONSTRUCTION, INC. STEEL DESIGN GUIDE SERIES 11: Floor Vibrations Due to Human Activity. 2003.

[2] ASSOCIAÇÃO BRASILEIRA DE NORMAS TÉCNICAS. NBR 6118: Projeto de estruturas de concreto - Procedimento. 2013.

[3] BATHE, Klaus-Jürgen. Finite Element Procedures. 14. ed. :Prentice Hall, 1996.

[4] CLOUGH, Ray W.; PENZIEN, Joseph. Dynamics of Structures. Mcgraw-Hill International Book Company, 1982.

[5] COMITÉ EURO-INTERNATIONAL DU BÉTON. BULLETIN D'INFORMATION N209: Vibration Problems in Structures. 1991.

[6] INTERNATIONAL STANDARDS ORGANIZATION. INTERNATIONAL STANDARD ISO 2631-2: Evaluation of Human Exposure to Whole-Body Vibration-Part 2: Human Exposure to Continuous and Shock-Induced Vibrations in Buildings (1 to $80 \mathrm{~Hz}) .1989$.

[7] VARELA, Wendell D.; BATTISTA, Ronaldo C.. Control of vibrations induced by people walking on large span composite floor decks. Engineering Structures. p. 2485-2494. set. 2011. 